\title{
Empleo y mercados de trabajo en la República Dominicana: una revisión de la literatura
}

\section{José R. Sánchez-Fung}

Departamento de Economía,

El presente artículo pasa revista a la literatura sobre el empleo, Universidad de Kent, Reino Unido, Reino Unido los salarios y los mercados de trabajo en la República Domijrs6@ukc.ac.uk nicana. Aunque la investigación interesará primordialmente a los economistas, puede ser útil también para los cientistas sociales en general y para quienes formulan políticas relativas al funcionamiento del mercado del trabajo tanto en el plano nacional como en la región latinoamericana. El estudio se inicia con la presentación de las principales fuentes de información estadística relacionadas con el trabajo; se centra después en las investigaciones sobre el funcionamiento global del mercado laboral dominicano. A continuación examina los trabajos que han explorado mercados laborales específicos, como el de las microempresas y el de las mujeres, y luego analiza la bibliografía sobre salarios. A partir de esa revisión se advierte que son pocos los estudios hechos con rigor científico y orientación económica: predomina el análisis económico descriptivo, sociológico o multidisciplinario. El artículo concluye con el planteamiento de posibles líneas de investigación futura en esta materia, principalmente el análisis de las estadísticas laborales existentes y la estructuración de un sistema apropiado para su recopilación y divulgación. 


\section{I}

\section{Introducción}

En el presente estudio se pretende organizar las investigaciones emprendidas con la finalidad de comprender el desenvolvimiento del factor trabajo y sus elementos asociados en la República Dominicana. Con este propósito, se exploran los trabajos sobre el empleo y el desempleo, los salarios y distintos mercados del trabajo. La investigación será de interés primordialmente para los economistas, pero los temas estudiados podrían ser de utilidad para cientistas sociales y diseñadores de políticas relacionados con el funcionamiento del mercado de trabajo, tanto en la República Dominicana como en América Latina.

El ensayo no es en su totalidad de naturaleza crítica. Hace hincapié principalmente en los estudios más importantes desde el punto de vista económico, aun- que se reseñan también trabajos provenientes de otras disciplinas o multidisciplinarios. De las investigaciones cuantitativas sólo se revisan detalladamente las más recientes, que son las de mayor alcance y cuyas bases de datos probablemente puedan ser consultadas en la actualidad.

Luego de examinar las estadísticas laborales en las principales fuentes de datos para el análisis del trabajo en la República Dominicana, se resumen los estudios que han analizado el empleo total. En seguida se reseñan los análisis de mercados de trabajo específicos. Después de examinar los principales estudios sobre los salarios, se concluye la investigación presentando recomendaciones sobre la dirección que podrían tomar trabajos futuros en esta materia.

\section{II}

\section{Las estadísticas laborales}

Las fuentes empleadas para estudiar el tema del trabajo en la República Dominicana han tenido distintas estructuras metodológicas y vigencia en el tiempo. No existe en el país una fuente que haya recolectado información sobre el trabajo sistemáticamente $y$ a través del tiempo, aunque se han hecho numerosos esfuerzos por lograr este objetivo.

\section{Las fuentes primarias}

Los censos de población levantados por la Oficina Nacional de Estadística (ONE) en 1920, 1935, 1950, 1960, 1970, 1981 y 1993 son la fuente de partida por exce-

El autor agradece el respaldo intelectual y los comentarios de José Luis Alemán, Rolando Guzmán, Amelia U. Santos de Sánchez y de dos jueces anónimos que formularon sugerencias sobre la forma y el contenido de versiones anteriores del trabajo. También desea reconocer los comentarios de Francisco Pérez Luna y Apolinar Veloz. La investigación ha sido posible, en parte, gracias al apoyo institucional de la Pontificia Universidad Católica Madre y Maestra (PUCMm), Santo Domingo, República Dominicana. Todos los errores que pueda contener el estudio son de su entera responsabilidad. lencia para el análisis de la evolución de la población en general y de ciertas características de los participantes en el mercado de trabajo, así como para el diseño de muestras de otros estudios de naturaleza similar, como las encuestas. ${ }^{1}$

Además de los censos, se han levantado encuestas en más de una ocasión. Entre ellas figura la encuesta demográfica y de salud (ENDESA), una investigación de carácter múltiple que se ha llevado a cabo en 1986, 1991 y 1996 (véase ONAPLAN/CESDEM/PROFAMILIA, 1997) y que recoge algunas características del trabajo de los entrevistados. La información proveniente del cuestionario de hogar ampliado de esta encuesta ha servido de base para analizar aspectos específicos del mercado laboral dominicano (véase Ramírez, 1993).

Actualmente las principales estadísticas sobre este mercado son las que emanan de la encuesta de fuerza de trabajo que efectúa desde 1986 el Banco Central de

\footnotetext{
${ }^{1}$ Véase una síntesis compacta y actualizada de la historia, metodología y organización de los siete censos realizados en el país por la oNE en Nicasio y De La Rosa (1998).
} 
la República Dominicana. ${ }^{2}$ Aunque al comienzo la encuesta se efectuaba cuatro veces al año, a partir de 1993 se realiza anualmente, por razones presupuestarias. La encuesta es elaborada por el Banco Central de la República Dominicana y no por la Oficina Nacional de Estadística (ONE), también principalmente por razones presupuestarias y de recursos humanos. Lo mismo ha sucedido en el pasado con otras encuestas, como las de hogares, y con las estadísticas de precios y las cuentas nacionales.

Las encuestas de fuerza de trabajo del Banco Central recopilan información sobre ocupados y desocupados, ingresos medios y horas trabajadas por deciles de ingresos. A partir de 1996 se amplió su alcance con el fin de recopilar información sobre el tamaño de las empresas a que pertenecen los trabajadores, así como sobre otros ingresos que perciben y que facilitan la clasificación de los ocupados según si trabajan en el sector formal o informal de la economía. La encuesta también investiga los ingresos provenientes de remesas familiares y jubilaciones.

Entre las principales investigaciones llevadas a cabo en una sola ocasión figura la encuesta nacional de mano de obra ENMO-93 (véase FUNDAPEC, 1992), que se limitó a recopilar información sobre las empresas de zonas francas, el turismo y la agroindustria, y sobre los estudiantes de cuarto año de bachillerato y de carreras técnicas. Para los mencionados sectores la encuesta registró i) las características del mercado laboral; ii) la existencia de mano de obra; iii) las necesidades futuras de mano de obra, incluidas las de capacitación; ${ }^{4}$ iv) las opiniones y expectativas de los estudiantes, trabajadores y empresarios con relación a la situación y perspectivas del mercado de trabajo, y v) el desarrollo de las zonas francas, la agroindustria y el turismo, así como la relación entre estos sectores y el desarrollo de la economía en su conjunto.

Recientemente, la Oficina Nacional de Estadística efectuó un censo de servidores públicos en la República Dominicana (oNE, 1998). Según el censo, es-

\footnotetext{
${ }^{2}$ Los detalles metodológicos de esa encuesta, como el diseño de la muestra y las definiciones utilizadas, pueden consultarse en BANCENT 1997 , pp. 9 a 24, y Gregory (1997, pp. 1 y 2).

${ }^{3}$ Llevada a cabo en 1991 como parte del programa de cooperación técnica para el fortalecimiento del sistema de enseñanza técnicovocacional en la República Dominicana., que realiza la Fundación APEC de Crédito Educativo Inc. (FUNDAPEC) con recursos del Banco Interamericano de Desarrollo.

${ }^{4}$ Las necesidades de capacitación fueron estimadas sobre la base de previsiones de la cantidad de trabajadores que anualmente se incorporarían a los sectores económicos analizados.
}

tos trabajadores representan el $11.64 \%$ de la población económicamente activa, con un total de $303478 \mathrm{em}$ pleados, de los cuales $41.1 \%$ son mujeres. Además, casi la mitad (40.8\%) tienen edades comprendidas entre los 35 y los 49 años. Una de las cifras más interesantes arrojadas por el censo es que sólo aproximadamente una quinta parte de los servidores públicos posee educación universitaria a nivel de grado (gráfico 1).

\section{Fuentes primarias de menor alcance}

Del Rosario e Hidalgo (1986) concibieron y aplicaron al caso dominicano una metodología para el cálculo de índices de salarios nominales y reales. Lamentablemente, no se continuó la recolección de esos índices.

En lo que concierne al sector rural, las características de la mano de obra fueron analizadas a partir de la encuesta elaborada en 1989 por la Unidad de Estudios Agropecuarios del Consejo Nacional de Agricultura (Santana, 1990).

De gran utilidad para el análisis de las microempresas y empresas pequeñas fueron las investigaciones del Fondo para el Financiamiento de la Microempresa (FONDOMICRO), que contienen, entre otras, estadísticas sobre el trabajo en ese sector (Cabal, 1992 y 1993; Moya y Ortiz, 1994 y 1995; Ortiz, 1996 y 1998).

También son de interés las estadísticas sobre distintos aspectos de los sindicatos en el país (ONE, 1993), y el Boletín de estadísticas laborales de la Secretaría de Estado de Trabajo (véase República Dominicana, Secretaría de Estado de Trabajo, 1999), que contiene cifras generadas tanto por esa dependencia como por otras instituciones.

GRAFICO 1

República Dominicana: Servidores públicos según nivel educativo, 1997

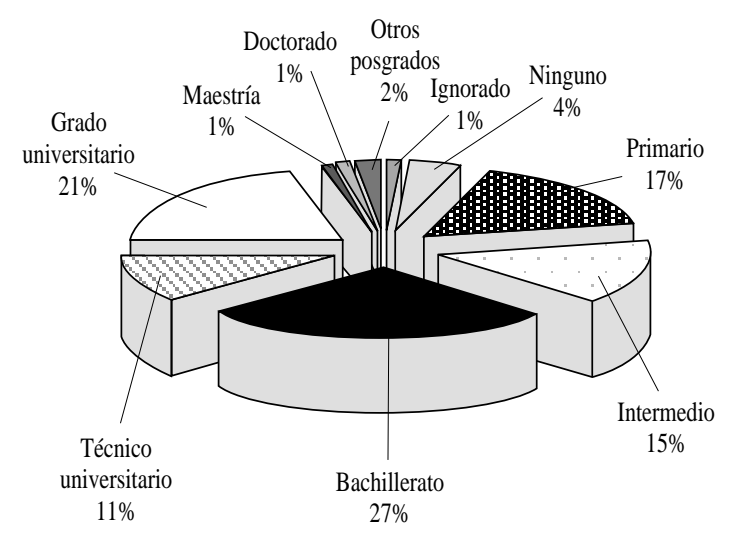

Fuente: ONE (1998). 
El Boletín de estadísticas laborales, que alcanza ya cuatro números, provee información detallada sobre el registro de empresas, nuevos empleos generados por sexo y monto pagado según rama de actividad, tamaño de empresa y régimen de producción y empleos ocasionales generados anualmente.

Las informaciones citadas son recogidas por la Secretaría de Estado de Trabajo a través de los formularios Planilla de personal fijo (DGT-3) y Cambios en la relación de personal fijo o de planillas (DGT-4), los que deben ser completados (según lo estipula el Código de Trabajo) por los establecimientos industriales, comerciales y de servicios registrados en el Departamento de Trabajo de dicha Secretaría.

Aunque la cantidad de datos disponibles en los boletines de la Secretaría del Estado de Trabajo no es suficiente para realizar análisis econométricos basados en series temporales, existe la posibilidad de acceder a las microfuentes originales y depurarlas, para elaborar estudios de corte tranversal o longitudinales. Sería interesante, además, que en el futuro las estadísticas generadas por esa Secretaría fueran comparadas con las producidas en la encuesta de fuerza de trabajo que efectúa el Banco Central.

La encuesta nacional de cultura política y democrática (DEMOS) podría aprovecharse como fuente de da- tos para el análisis de las relaciones laborales (véase Duarte Brea y Tejada, 1998), mientras que la encuesta nacional de jóvenes de 15 a 24 años (ENJOvEN-92) proporciona datos sobre la participación de este grupo en el mercado de trabajo (Tejada, Herold y Morris, 1998).

Existen también fuentes de información estadística sobre dos de los sectores más dinámicos de la economía dominicana: las zonas francas y el turismo. Las publicaciones periódicas del Consejo Nacional de Zonas Francas de Exportación informan sobre las zonas francas e incluyen datos sobre el empleo.

Para obtener informaciones históricas sobre el empleo en el sector del turismo es útil consultar $T u$ rismo en cifras (República Dominicana, Secretaría de Estado de Turismo, 1996).

Otras encuestas relacionadas con el factor trabajo que han sido levantadas en el país son la de la Oficina Nacional de Planificación (véase ONAPLAN, 1968), la del Banco Central de la República Dominicana, de la Oficina Nacional de Estadística y la Agencia de los Estados Unidos para el Desarrollo Internacional (BANCENT/ONE/USAID, 1968) y la de esta misma agencia con la Secretaría del Estado de Agricultura (USAID/ República Dominicana, Secretaría de Estado de Agricultura, 1977). ${ }^{5}$

\section{III}

\section{Análisis global del mercado laboral}

Gran parte de las investigaciones sobre el mercado de trabajo dominicano se fundamentan en análisis de encuestas, la mayoría sin continuidad, lo que limita la posibilidad de llevar a cabo comparaciones históricas sistemáticas entre el desarrollo de distintas variables o relaciones.

\footnotetext{
${ }^{5}$ Además de las analizadas, existen fuentes secundarias que contienen estadísticas que han sido o pueden ser utilizadas para estudiar diversos elementos ligados al trabajo en la República Dominicana. Entre ellas cabe citar Ramírez, Tatis y Germán (1983) y Quiterio y Polanco (1990), quienes elaboran proyecciones de la población y la mano de obra para diversos períodos, basándose principalmente en los censos de población. De igual importancia son las series de tiempo sobre el salario mínimo nominal y real en los sectores público y privado, así como las estadísticas e indicadores del personal ocupado del gobierno central recolectadas por Adolfo Martí (1997, pp. 339-347). Por último, el Anuario de estadísticas del trabajo publicado por la OIT (por ejemplo, оIт,1998) es una fuente importante de datos.
}

A manera de ilustración, el gráfico 2 muestra la magnitud del desempleo en la República Dominicana en el período 1968-1998, según estimaciones obtenidas a partir de varias fuentes. ${ }^{6}$ Aparentemente el fenómeno persiste durante todo el período en cifras que van del 15 al 28\%, aproximadamente, aunque no muestra una tendencia temporal ni volatilidad pronunciadas.

De los múltiples estudios del empleo que se han hecho en el país sólo unos cuantos pueden considerarse suficientemente rigurosos y, por lo tanto, de utilidad para fines de referencia. Esta observación es aún válida para las investigaciones efectuadas una o más décadas atrás.

\footnotetext{
${ }^{6}$ No todos los datos del gráfico 2 provienen de fuentes comparables, por lo cual deben ser analizados con precaución.
} 
GRAFICO 2

República Dominicana: Desempleo, 1968-1998

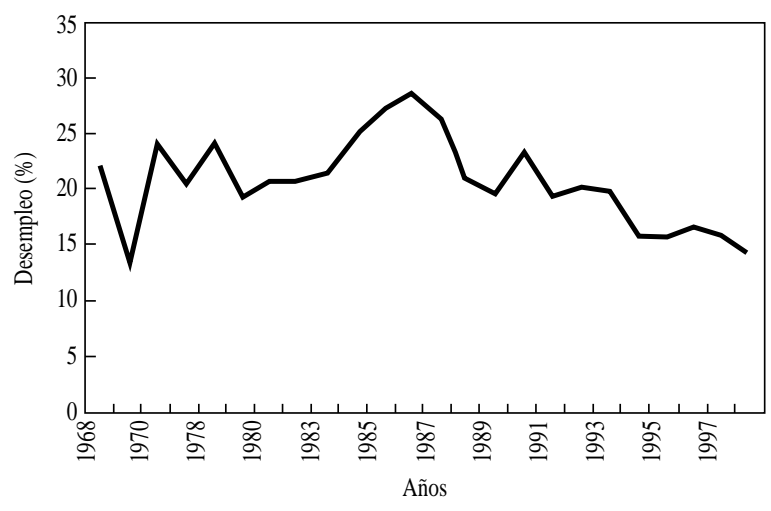

Fuente: Para los años 1968-1980 y 1982-1983, García y Valdivia 1985, cuadro 24, p. 65); para los años 1981 y 1984-1990, Ceara (1992, cuadro 1, p. 14) y para el período 1991-1998, datos del Banco Central de la República Dominicana.

El informe Generación de empleo productivo y crecimiento económico, el caso de la República Dominicana (оIт, 1975) puede considerarse el más completo y citado en su clase; comprende tanto análisis microeconómicos como macroeconómicos. ${ }^{7}$ Luego de una sección inicial de resumen y conclusiones, el texto principal se divide en cinco grandes partes: desempleo y pobreza: la situación actual; tendencias recientes de la oferta y la demanda de mano de obra; el empleo en tres sectores básicos, y factores institucionales; además tiene algunos anexos.

El estudio se fija como uno de sus principales objetivos estimar el impacto de un crecimiento económico acelerado sobre el empleo y, a partir de esa relación, analizar la forma en que el desarrollo pudiera desembocar en un aumento del empleo El análisis intenta cumplir con esa tarea penetrando tanto en el campo de la economía positiva como en el de la normativa.

Una deficiencia de la investigación radica en que la gran cantidad de análisis estadísticos presentados, de los cuales depende la mayoría de sus conclusiones, son básicamente descriptivos. El estudio no aplica técnicas econométricas sobre hipótesis particulares que puedan respaldar científicamente sus conclusiones. Además, como el mismo estudio señala, las cifras empleadas deben ser consideradas con precaución.

\footnotetext{
${ }^{7}$ Una versión de ese informe fue publicada por la Oficina Nacional de Planificación (ONAPLAN, 1974).
}

Una de las principales conclusiones del análisis es que "Desde 1969, la economía dominicana crece al ritmo extraordinariamente rápido de 10.9 por ciento al año, ...Sin embargo, la rápida expansión económica no parece haber reducido el desempleo en Santo Domingo" (оIт, 1975, p. 5). Como se verá más adelante, esta situación persiste y plantea interrogantes a las autoridades gubernamentales, a los economistas y a los cientistas sociales en general.

García y Valdivia (1985) efectuaron un análisis general auspiciado por la ort de la situación de la economía dominicana en 1980-1983, que abarca con cierto detalle el tema del empleo. Específicamente, los autores analizan el proceso de ajuste de la economía dominicana a la crisis internacional de $1980-1983$ y sus repercusiones sobre el mercado laboral. Para los fines de la presente investigación, la sección titulada "El empleo y los ingresos" es de particular interés. En ella los autores analizan el crecimiento de la fuerza de trabajo, el empleo y el desempleo en áreas urbanas, la evolución de los salarios reales a mediano plazo y el ajuste del mercado de trabajo en 1980-1983.

Igual que la oाт, García y Valdivia (1985, pp. 67 y 68) destacan que "un rasgo estructural presente aun en el período de mayor dinamismo de la economía dominicana es la tendencia a una elevada subutilización de la fuerza de trabajo, tanto en las áreas rurales como urbanas, manifestada en las elevadas tasas de desempleo abierto y subempleo detectadas por diversas fuentes ... la crisis 1980-1983 se sobreimpone a tendencias ya arraigadas en el desarrollo de República Dominicana, acentuando los ya serios problemas que el país enfrentaba".

Los autores señalan además las tendencias de los salarios reales a disminuir durante 1974-1983, observando que esas reducciones ocurrieron a pesar de los subsidios aplicados a varios componentes del índice de precios al consumidor durante gran parte del período citado. La investigación también revela que las diferencias de ingresos entre Santo Domingo y los demás centros urbanos del país tendieron a aumentar durante el período.

Recientemente, Gregory hizo un estudio del mercado de trabajo dominicano, basado principalmente en la encuesta de fuerza de trabajo que elabora el Banco Central, aunque sin limitarse a esa fuente (Gregory, 1997). ${ }^{8}$ En él analiza tanto la estructura como de los

\footnotetext{
${ }^{8}$ Es interesante que la génesis del trabajo de Gregory haya sido el comentario de Arnold Harberger (1997, pp. 12-14) respecto al crecimiento económico y el nivel de desempleo de la economía domi-
} 
resultados de las encuestas del Banco Central. Luego de examinar la metodología de tales encuestas, llega a la conclusión de que las altas tasas de desempleo arrojadas por éstas se deben, al menos en parte, a la medida de desempleo utilizada: esas encuestas clasifican como desempleadas a personas que no han buscado activamente un puesto de trabajo durante un período razonable, pudiendo así ser clasificada como desempleada una persona con varios meses de búsqueda pasiva.

En el cuadro 6 de la obra citada Gregory presenta distintos escenarios, basándose en las declaraciones de los desempleados sobre la búsqueda de empleo. La diferencia entre las medidas es considerable. Sobre la base de los datos de la muestra ampliada de la encuesta del Banco Central para abril del 1997, la tasa de desempleo era de $15.7 \%$ si se registraba como desempleados a aquellos que declararon que aceptarían un trabajo si se les ofreciera, mientras que el mismo indicador para los que buscaban trabajo activamente (menos de un mes de búsqueda) era de $6.5 \%$.

En el pasado también se habían hecho estimaciones del desempleo sobre la base del tiempo de búsqueda. García y Valdivia (1985, cuadros 24 y 25) ajustan una serie de la variable desempleo obtenida a partir de varias encuestas. Las medidas de desempleo, como es de esperarse, variaron al excluir de los desocupados abiertos y de la población económicamente activa a todos aquellos que declaraban haber estado buscando trabajo por un año o más en el período de referencia de la encuesta. A manera de ilustración puede señalarse que la tasa de desocupación se redujo de $24 \%$ a $20.8 \%$ (ajustada) en 1970, y de $21.4 \%$ a $15 \%$ (ajustada) en 1983. Hubiera sido interesante que los autores también hubiesen calculado tasas de desempleo partiendo de períodos de búsqueda menores, como lo hizo el Banco Central.

De las investigaciones revisadas hasta este punto se infiere que el conocimiento existente sobre el mercado laboral dominicano es en gran medida incierto.
Los principales estudios (orT, 1975; García y Valdivia, 1985; Gregory, 1997) basan sus análisis sobre datos estadísticos no del todo confiables, además de no contener componentes científicamente rigurosos que extraigan el mayor provecho de ellos.

Márquez (1998), por ejemplo, señala la heterogeneidad, en términos de magnitud, del desempleo en América Latina, por un lado, y en el Caribe, por otro, siendo históricamente más bajo en la primera. Sin embargo, el autor indica que estudios recientes como el de Rama (1995) para Jamaica y de Gregory (1997) para la República Dominicana señalan la posibilidad de que las altas tasas de desempleo registradas en estos países estén afectadas por problemas de medición.

Las fuentes primarias de estadísticas del país pocas veces han tenido continuidad, y hasta ahora una sola, la encuesta de fuerza de trabajo del Banco Central, está diseñada específicamente para recoger información sobre el mercado laboral.

¿Qué puede decirse acerca del comportamiento global del mercado laboral dominicano? Citando a Gregory (1997) cabe señalar que "el consenso popular en lo relativo a las condiciones de empleo en la República Dominicana es de que son terribles. El desempleo afecta a entre el 20 y el $60 \%$ de la fuerza de trabajo, siendo $30 \%$ cercano al valor modal. Citar cifras de desempleo a estos niveles continuamente en los últimos 30 años o más se ha vuelto un dogma de fe. En este punto de vista existe una diferencia conceptual notable entre la visión pública y la de los economistas especializados en cuestiones laborales sobre qué constituye el desempleo. De lo que pudimos obtener de conversaciones y otras fuentes, la visión del público es que cualquiera que no sea empleado de una empresa moderna o del gobierno es un desempleado. Claramente, esto se aleja mucho de la definición estándar de desempleo, un estatus asignado a los individuos que no tienen un empleo remunerado y que están buscando trabajo activamente" (Gregory, 1997, p. 33). nicana. Harberger señaló que las saludables tasas de crecimiento del país no correspondían al alto nivel de desempleo registrado por las encuestas del Banco Central. Observaciones similares aparecen en trabajos como огт (1975), García y Valdivia (1985), Cabral (1975), Duarte (1983), Lozano (1987), Liz (1993), Weller (1993 y
1998), Alemán (1994) y Vargas (1995). Además, análisis como los de Alemán (1996, a y b), Aristy (1996) y Santana (1996) plantean estrategias específicas para elevar la capacidad de generación de empleo de la economía dominicana, dada la aparente persistencia de la situación descrita por Harberger y muchos otros autores. 


\section{IV}

\section{Mercados de trabajo específicos}

\section{Las microempresas}

Ciertas características del empleo en el sector de las microempresas y empresas pequeñas —uno de los más dinámicos de la economía dominicana en el pasado reciente - han sido analizadas detalladamente en varios estudios. La participación de las mujeres en ese sector fue investigada por Cely (1993), sobre la base de la primera encuesta nacional de las microempresas y empresas pequeñas realizada por FONDOMICRO en marzo de 1992.

Cely analiza características específicas de esas empresas, como la clase de trabajadoras que las componen, su estructura, las actividades a las cuales se dedican y los métodos de financiamiento. Entre los hallazgos más interesantes de la investigación se encuentra el hecho de que al momento de efectuarse la encuesta alrededor del $45.8 \%$ de las empresas del sector eran propiedad de mujeres, aunque sólo generaban el $33.4 \%$ del empleo en el sector.

También basándose en las encuestas elaboradas por FONDOMICRo, Cabal (1996) analiza los cambios registrados en el tamaño del empleo en el sector de las microempresas y pequeñas empresas entre 1992 y 1995. Define el cambio en el empleo agregado del sector en un período de tiempo dado como el resultado de dos componentes básicos, a saber, el cambio neto en el empleo originado por las empresas que nacen y por las que cierran, y el cambio en el empleo proveniente de la expansión neta de empresas que sobreviven. Esta definición se expresa en la ecuación [1].

$$
\frac{\Delta e}{e_{t}}=\left[\frac{e n}{e_{t}}-\frac{e m}{e_{t}}\right]+\left[\frac{\Delta e p^{x}}{e_{t}}-\frac{\Delta e p^{c}}{e_{t}}\right]
$$

en que en indica el número de empleos generados por empresas nuevas, em los empleos perdidos debido al cierre de empresas, $\Delta e p^{x}$ el incremento en el empleo de las empresas que se expanden, $\Delta e p^{c}$ el cambio en el empleo de las empresas que se han contraído, mientras que $e_{t}$ es el empleo total del sector en el período $t$.

A partir de la fórmula [1], Cabal estima que el crecimiento neto del empleo en el sector de las microempresas y empresas pequeñas durante los períodos 1992-1993, 1993-1994 y 1994-1995 fue de 8\%,
$1.5 \%$ y $0.6 \%$, respectivamente. Los resultados del análisis parecen indicar que el empleo en el sector se ha expandido más cuando la economía ha crecido menos y viceversa, es decir, que la variable presenta un comportamiento contracíclico (gráfico 3). Sin embargo, esa conclusión debe aceptarse con cautela ya que está fundamentada en el análisis de escasas observaciones de las variables.

Cely (1996), igual que Cabal, empleó la fórmula [1], pero aplicada a microempresas y empresas pequeñas de mujeres durante el período 1992-1995. Obtuvo resultados distintos, ya que el empleo en esas empresas de mujeres se redujo en $-1.4 \%,-0.9 \%$ y $-8.8 \%$ en los períodos 1992-1993, 1993-1994 y 1994-1995, respectivamente. En las empresas de hombres, en cambio, el empleo neto aumentó en $16.5 \%$, $8.7 \%$ y $3.9 \% .^{9}$

Recientemente, Ortiz (1998) exploró algunas características de las microempresas y su fuerza laboral. Según ese estudio, en 1998 los trabajadores de estas empresas representaban un $28.4 \%$ de la población eco-

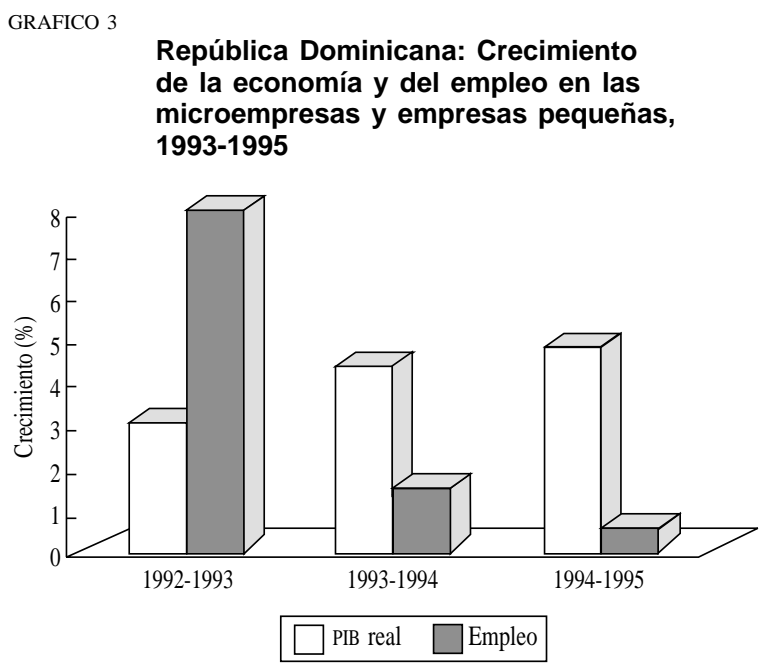

Fuentes: Banco Central de la República Dominicana y Cabal (1996).

\footnotetext{
${ }^{9}$ En Cely (1996, capítulo 5) se da una explicación detallada del comportamiento de cada uno de los componentes involucrados en el cálculo de la variación del empleo neto en las microempresas y empresas pequeñas, clasificadas por sexo, durante los períodos señalados.
} 
nómicamente activa (es decir, 955683 empleados) con una edad media de 28 años y una distribución equilibrada en cuanto al género. La investigación además informa que, en promedio, los empleados de las microempresas y empresas pequeñas encuestadas poseen una formación académica más elevada que los propietarios.

\section{Las mujeres}

Una fuente importante de partida para el análisis del trabajo de la mujer en la República Dominicana es la bibliografía elaborada por Sherrie Baver (The CUNY Dominican Studies Institute, 1997, pp. 33-40), que señala los principales estudios, investigadores y centros de investigación que han analizado la integración de la mujer al mercado del trabajo dominicano. Además, el estudio de Jean Weisman (The cuny Dominican Studies Institute, 1997, pp. 41-56) analiza importantes aspectos del trabajo de la mujer en el país.

Gurak, Kritz, Mota y Ortega (1979) presentan una investigación del empleo femenino en la República Dominicana donde analizan en particular el importante efecto de la fecundidad sobre la participación de la mujer en el mercado laboral. El estudio muestra que, entre las mujeres casadas, la fecundidad se relaciona fuertemente con la educación y la ocupación: es negativa respecto de la primera y ambigua con relación a la segunda. Para el universo de mujeres empleadas, el estudio encuentra que la probabilidad de empleo está relacionada positivamente con la educación tanto de la entrevistada como de sus padres, y negativamente con la edad y el estar casada. La educación, sin embargo, se relaciona negativamente con el trabajo en servicios, en general, y el servicio doméstico, en particular. Para el caso de las mujeres no empleadas, tanto la intención de trabajo futuro como la búsqueda de trabajo están relacionadas positivamente con la educación del padre y negativamente con la existencia de una unión sentimental.

En Forum 21 (Moya, ed., 1986) se analizan algunos aspectos de las condiciones socioeconómicas de la mujer trabajadora en la República Dominicana desde un punto de vista básicamente sociológico. Entre los trabajos que allí figuran se encuentra un conjunto de estadísticas presentadas por Clara Báez (pp. 49-55), referentes a la participación de la mujer dominicana en el mercado de trabajo. Esas estadísticas indican que el sector laboral femenino más importante del país es el de las trabajadoras domésticas. Las mujeres están en desventaja en cuanto a salario y jerarquía con relación al hombre, tienen escasa participación en el mercado laboral rural agropecuario, y, en cambio, predominan en el rural no agropecuario.

El compendio Forum 21 incluye, además, un trabajo de François Pou (pp. 83-107) que examina la encuesta nacional de mujeres rurales levantada entre diciembre de 1984 y enero de 1985 por el Centro de Investigación para la Acción Femenina (CIPAF). Pou llega a la conclusión de que en el sector rural la mujer tiende a orbitar alrededor de las actividades relacionadas con el hogar y, en menor magnitud, a ofrecer su mano de obra en el mercado laboral.

En los estudios preparados por Báez y Matrillé (1994) y por Martínez (1994) se investigan, por medio de información generada a partir de encuestas individuales, algunos aspectos del mercado laboral para las mujeres formadas en ocupaciones no tradicionales, ${ }^{10}$ sobre todo en el caso del sector Los Mina Norte de la ciudad de Santo Domingo.

Martínez señala que, a pesar de las barreras materiales y sociales que se oponen a la participación de las mujeres en oficios tradicionalmente reservados para hombres, éstas están dispuestas, de ser necesario, a ejecutar ese tipo de tareas. Sin embargo, para que esa participación sea efectiva deben cambiar las creencias muy arraigadas de las mujeres, y de la sociedad en su conjunto, sobre el carácter masculino de las ocupaciones en cuestión. Por su parte, Báez y Matrillé abogan por una mayor capacitación de las mujeres en ocupaciones no tradicionales, lo cual debe estimular un aumento de su participación en esos oficios.

\section{Trabajo de menores}

El trabajo de menores ha sido estudiado por varios autores, entre otros Duarte (1979), Duarte, Gómez y Ariza (1991), Lizardo (1994), Silié (1996) y Alemán (1996a). Alemán presenta una síntesis de los principales estudios realizados y analiza aspectos particulares, siendo uno de ellos el cambio en la estructura socioeconómica de los hogares. Alemán señala que el aumento en la participación de menores en el mercado de trabajo ha estado acompañado de una disminución en la participación de los adultos (véase Alemán 1996a, cuadro 19, p. 65$){ }^{11}$

\footnotetext{
${ }^{10}$ Según el criterio adoptado en esas investigaciones, serían oficios no tradicionales para la mujer, por ejemplo, la albañilería, la ebanistería y la mecánica automotriz.

${ }^{11}$ De 45.2 a $43 \%$ y de 95.3 a $89.8 \%$ entre 1981 y 1991 , para las jefas y jefes de familia, respectivamente.
} 
Duarte, Gómez y Ariza (1991) presentan estadísticas que muestran que la principal causa del trabajo de menores es la precariedad de sus hogares y no el simple deseo de sus padres (véase en ese trabajo el cuadro 4.5, p. 131).

\section{Zonas francas}

Varios estudios han investigado el desenvolvimiento del trabajo en las zonas francas industriales de exportación. Dauhajre, Riley, Mena y Guerrero (1989, pp. 67-109) ${ }^{12}$ han analizado el impacto económico de las zonas francas sobre el empleo en la República Dominicana. Además, con un análisis descriptivo de las estadísticas del empleo directo e indirecto generado y los salarios pagados, el estudio presenta estimaciones econométricas según las cuales una devaluación de la tasa de cambio oficial tendría un impacto positivo sobre el empleo en el sector, mientras que un aumento del salario mínimo causaría el efecto inverso.

Sin embargo, Gregory (1997, pp. 24-25) señala que "Las empresas de zonas francas también reportan ingresos que sobrepasan ampliamente los salarios mínimos establecidos para el sector. El promedio de ingresos mensuales para las mujeres, RD $\$ 2590$, en la primera mitad del 1996, representa un 54\% por encima del mínimo legal, mientras que en el caso de los hombres, RD\$ 3624 , éstos más que duplicaron el mismo".

Con un enfoque multidisciplinario, Pérez Sáinz (1995a) desarrolla un análisis comparado de la forma en que interactúan los gobiernos, empresarios y sindicalistas en relación con los problemas laborales en las maquilas y las zonas francas de Costa Rica y la República Dominicana. Además, el mismo Pérez Sáinz (1995b) compara diversos factores relacionados con las maquilas y las zonas francas en Centroamérica y la República Dominicana y, específicamente, aspectos ligados al proceso de generación de empleo por esas actividades.

\section{Migrantes y mano de obra haitiana}

Una de las características del mercado laboral dominicano ha sido la migración. El componente más importante es la migración de dominicanos hacia los Estados Unidos, generalmente a través de Puerto Rico. Sobre este tema Rodríguez (1999) señala que "Según cifras del Servicio de Inmigración y Naturali-

12 Véase también Corten y Duarte (1986). zación de los Estados Unidos, la República Dominicana, de poco más de siete millones de habitantes en 1990, aparecía en la lista de los diez países del mundo con un mayor número de inmigrantes admitidos como residentes permanentes en el período 1981-1996... En términos relativos, el número de dominicanos admitidos era mucho más significativo que el de los mexicanos; los primeros representaban el $7 \%$ de la población de la isla en 1990, mientras que los segundos alrededor del 4\% de la población de México".

Ramón Hernández y Narey López (The cunY Dominican Studies Institute, 1997, pp. 59-78) presentan una excelente visión del problema y una bibliografía completa de lo que se ha escrito sobre el tema.

La inmigración de mano de obra haitiana, por su parte, se ha dirigido principalmente a las zonas de cultivo de caña de azúcar y al sector de la construcción. También es significativa la cantidad de haitianos que se dedican a comercializar en la frontera con la República Dominicana. Según estimaciones de la Dirección General de Migración, más de 20000 haitianos entran y salen del país diariamente con fines de comercio (Listín Diario, 1999, p. 20A).

Respecto de la inmigración haitiana interesa averiguar cuál es su magnitud. ¿Qué cantidad de haitianos trabajan o viven (mano de obra potencial) en territorio dominicano? Corten y Duarte (1994) proveen abundante bibliografía sobre la materia. Según ellos, en la República Dominicana vive alrededor de medio millón de haitianos y dominicanos de origen haitiano, aunque la composición de la cifra no está claramente determinada.

También sería interesante saber si la mano de obra haitiana es desplazadora de la dominicana, o es muestra del libre juego de la oferta y la demanda de trabajo. La última opción parece razonable si se toma en cuenta que los dominicanos históricamente han repudiado el tipo de trabajo que ejecutan los haitianos, por ejemplo, el corte de la caña. El problema es complejo y ha sido analizado desde diversas perspectivas (Martínez, 1999).

Desde el punto de vista económico, el problema de la (in)migración puede ser razonablemente aproximado, en principio, a través de un modelo como el propuesto por Harris y Todaro (1970).

Aunque los problemas descritos son de importancia para el desarrollo de la economía y de la sociedad dominicanas, no existen estudios de naturaleza económica que analicen rigurosamente su dinámica. En cambio, son numerosas las investigaciones de otras ramas de las ciencias sociales (generalmente de la sociología). 


\section{V}

\section{Salarios}

\section{Ingreso y escolaridad}

La relación entre la educación y el mercado de trabajo en la República Dominicana ha sido analizada en muchos estudios. Entre ellos cabe mencionar Bases para formular una política de empleo en la República Dominicana (ONAPLAN, 1974) y Los recursos humanos y el empleo de la República Dominicana (Education Development Center, 1975). ${ }^{13}$

Sobre la relación específica entre ingresos y escolaridad, Santos (1998) presenta estimaciones econométricas basadas en datos de corte transversal provenientes de las encuestas demográficas y de salud de 1991 y $1996 .{ }^{14}$ El estudio analiza el impacto de la educación secundaria sobre el ingreso, tomando en consideración otros detalles como el tipo de escuela a la que asistió el individuo, el área geográfica de residencia, el género, el nivel de ingreso y la clase social.

Según los resultados obtenidos por la investigación, análogos para 1991 y 1996, hay una relación positiva entre el grado de escolaridad y el ingreso, siendo el haber estudiado en una escuela pública el único de los factores considerados que impacta negativamente en el ingreso. Por el contrario, la evidencia empírica de la investigación indica que residir en la zona urbana, ser de clase media o alta y ser hombre influyen positivamente en el ingreso del individuo.

\section{Salarios mínimos ${ }^{15}$}

Entre las escasas investigaciones relacionadas con los salarios mínimos en la República Dominicana figura la presentada recientemente por la Secretaría de Estado de Trabajo (República Dominicana, Secretaría de Estado de Trabajo, 1997). Esta investigación analiza la evolución de los salarios mínimos, la relación entre los pagados a hombres y mujeres y la relación de ese salario con varios agregados macroeconómicos de la economía dominicana. Indica, además, que el salario mínimo se paga a sólo al $4.1 \%$ de los ocupados.

Los autores concluyen que el salario mínimo aparentemente no tiene efectos en el nivel de inflación y que podría tener un impacto de importancia en el sector de servicios, por su elevada participación en la estructura de costos del sector.

Dauhajre, Achecar y Swindale (1994) también investigan la relación entre los salarios mínimos y la distribución del ingreso. Los autores concluyen que el salario mínimo real tiene un efecto positivo en el bienestar general, ya que al aumentar aparentemente mejora la distribución de la riqueza, medida aplicando el coeficiente de Gini.

Dauhajre, Riley, Mena y Guerrero (1989, pp. 67109) presentan estimaciones econométricas según las cuales una devaluación del tipo de cambio oficial tendría un impacto positivo sobre el empleo en el sector, mientras que un aumento del salario mínimo causaría el efecto inverso. La explicación radicaría en que la devaluación, en igualdad de condiciones, reduce el costo de la mano de obra local expresada en moneda extranjera.

Sin embargo, como se dijo más atrás, Gregory (1997, pp. 24-25) señala que los salarios de las empresas de zonas francas sobrepasan ampliamente los salarios mínimos establecidos para el sector (en 54\% para las mujeres y más de $100 \%$ para los hombres en la primera mitad de 1996). Asevera además (ibid., p. 21), que la multiplicidad de salarios mínimos en la economía dominicana complica cualquier esfuerzo encaminado a cuantificar el impacto que tiene el salario mínimo legal sobre los ingresos reales de los trabajadores.
13 Cabral (1976) analiza esos estudios.

14 Los determinantes de los niveles de escolaridad alcanzados en la República Dominicana fueron analizados por Lizardo y Guzmán (1999).

\footnotetext{
15 Véase más atrás la sección IV, subsección 4, donde se presentan algunos aspectos de los salarios mínimos en las zonas francas.
} 


\section{VI}

\section{Conclusión y posibles líneas de investigación futura}

El objetivo principal del presente estudio ha sido revisar la literatura sobre el trabajo en la República Dominicana. Aunque el análisis ha identificado una cantidad significativa de investigaciones, es notable la escasez de trabajos elaborados con orientación económica y rigurosidad científica, prevaleciendo el análisis económico descriptivo, el sociológico, o el multidisciplinario.

Las investigaciones futuras en la materia podrían encaminarse, entre otras direcciones, a la elaboración de estudios econométricos que contribuyan a verificar la consistencia de las estadísticas disponibles en la actualidad (primordialmente la encuesta de fuerza de trabajo del Banco Central).

Sería interesante, con un análisis de ese tipo, intentar dar respuestas rigurosas a preguntas como las siguientes: ¿Existe realmente desempleo en proporciones significativas? ¿Cuál es su incidencia? ¿Qué tipo de relación hay entre las vacantes y el desempleo? ¿Cuál es la duración media de los períodos de desempleo? ¿Cuáles son las tasas de entrada y salida del desempleo? ¿Cómo impactan los salarios mínimos en el desarrollo de la economía o de sectores específicos? ¿Cuáles son los factores determinantes de la inversión en capital humano? ¿Cómo afecta el desarrollo tecnológico al bienestar de los trabajadores? ¿Hay diferencias notables entre los salarios pagados a trabajadores con destrezas similares por el simple hecho de que laboren en industrias diferentes? ¿Qué papel desempe- ña el trabajo de menores? ¿Hay desigualdades relativas entre las oportunidades de trabajo para las mujeres y los jóvenes? ¿Cuál es el papel del gobierno como empleador? ¿Cómo se determinan los salarios y el empleo en el sector público?

Los resultados de estudios por esas líneas podrían ser utilizados por los encargados de diseñar y aplicar políticas relacionadas con el factor trabajo en el país y, además, en el mejoramiento de la recolección de las estadísticas. Es imprescindible la estructuración de un sistema capaz de recopilar estadísticas laborales que sean confiables y, sobre todo, accesibles periódicamente.

Hay otras líneas de investigación que podrían resultar fructíferas, como el análisis detallado de una serie de factores institucionales, por ejemplo, las consecuencias económicas del actual código laboral dominicano (Ley 16-92). ${ }^{16}$ Además, los estudios de las diversas formas en que interactúan los sindicatos y los patrones en la determinación del nivel de empleo y los salarios son casi inexistentes en la República Dominicana. ${ }^{17}$

No menos importante es el tema de la estructuración de un sistema de seguridad social sólido y, sobre todo, autosustentable en el tiempo. El componente capital de un sistema de esa naturaleza, una legislación adecuada, está siendo debatido en la actualidad por el Congreso Nacional (véase Senado de la República Dominicana, 1999). ${ }^{18}$

\section{Bibliografía}

Alemán, J. L. (1994): Empleo y apertura externa en la República Dominicana, Estudios sociales, $N^{\circ}$ 97, Santo Domingo, Centro de Investigación y Acción Social de la Compañía de Jesús.

\footnotetext{
${ }^{16}$ Dauhajre, Aristy, Riley, Mena, Féliz, Guiliani y De Castro (1996, pp. 459-472) han hecho un análisis compacto de ese código.

${ }^{17}$ No obstante, son de utilidad los trabajos de De Peña (1977) y Cassá (1990). El primero resume temas históricos de relevancia para el análisis del movimiento sindical en la República Dominicana, mientras que el segundo desarrolla un análisis histórico-sociológico de los movimientos obreros. Hernández (1998) aborda el tema de
}

(1996a): Políticas y programas sociales: menores en dificultad, generación de empleo y pobreza crítica, Santo Domingo, Pontificia Universidad Católica Madre y Maestra/ Centro Universitario de Estudios Políticos y Sociales (PUCMM/ CUEPS)/Editora Centenario.

la negociación colectiva en la República Dominicana desde el punto de vista jurídico.

${ }^{18}$ Véase también, a manera de ilustración, Santana y Rathe (1993), PUCMM/CUEPS (1996, capítulo VI, pp. 327-368) y Hernández y Hernández (eds., 1996); el último trabajo trata primordialmente los aspectos jurídicos del tema. 
(1996b): Lucha contra la pobreza, enfatizando la generación de empleo, PUCMM/CUEPS, Agenda nacional de desarrollo. Planes de acción para las diez principales prioridades, vol.II, Santo Domingo, Grupo de Acción por la Democracia (GAD)/Editora Centenario.

Aristy, J. (1996): Políticas encaminadas a la reducción de la pobreza y a la generación de empleo, PUCMM/CUEPS, Agenda nacional de desarrollo. Planes de acción para las diez principales prioridades, vol. II, Santo Domingo, GAD/Editora Centenario.

Báez, C. y M. Matrillé (1994): Mercado laboral para las mujeres formadas en ocupaciones no tradicionales: Caso Los Mina Norte, Santo Domingo, Banco Interamericano de Desarrollo (BID)/Fundación APEC de Crédito Educativo, Inc. (FUNDAPEC)/ Editora Palma.

BANCENT/ONE/USAID (Banco Central de la República Dominicana)/ Oficina Nacional de Estadística/Agencia de los Estados Unidos para el Desarrollo Internacional) (1968): Estudio sobre presupuestos familiares, Santo Domingo.

BANCENT (Banco Central de la República Dominicana)(1997): Mercado de trabajo 1991-1996, Santo Domingo.

(varios años): Boletín trimestral, varios números, Santo Domingo.

Cabal, M. (1992): Microempresas y pequeñas empresas en la República Dominicana, resultados de una encuesta nacional, Santo Domingo, Fondo de Desarrollo para la Microempresa (FONDOMICRO).

(1993): Evolución de las microempresas y pequeñas empresas en la República Dominicana, 1992-1993, Santo Domingo, FONDOMICRO.

(1996): Cambios en el tamaño y el empleo de las microempresas y pequeñas empresas en la República Dominicana, 1992-1995, Santo Domingo, FONDOMICRO.

Cabral, M. J. (1975): La inflación dominicana, distribución del ingreso y empleo, Academia de Ciencias de la República Dominicana, Anuario, vol. 1, pp. 431-462, Santo Domingo, Instituto Tecnológico de Santo Domingo.

(1976): Algunas observaciones sobre la educación y el empleo en la República Dominicana, Eme Eme, N 5 , Santo Domingo.

Cassá, R. (1990): Movimiento obrero y lucha socialista en la República Dominicana, desde los orígenes hasta 1960, Santo Domingo, Fundación Cultural Dominicana/ Editora Taller.

Ceara, M. (1992): Empleo, deuda y crecimiento económico: un modelo macro, Ensayos de Economía, Santo Domingo, Centro de Investigación Económica para el Caribe (CIECA), abril.

Cely, P. (1993): Microempresas y pequeñas empresas de mujeres en la República Dominicana: resultados de una encuesta nacional, Santo Domingo, FONDOMICRO.

(1996): Dinámica de las microempresas y pequeñas empresas de mujeres en la República Dominicana, 1992-1995: una perspectiva dinámica, Santo Domingo, FONDOMICRO.

Corten, A. e I. Duarte (1986): Procesos de proletarización de mujeres: las trabajadoras de industrias de ensamblajes, en I. Duarte (ed.), Trabajadores urbanos: ensayos sobre fuerza laboral en la República Dominicana, Estudios sociales, Santo Domingo, Centro de Investigación y Acción Social de la Compañía de Jesús.

(1994): Quinientos mil haitianos en República Dominicana, Estudios sociales, $\mathrm{N}^{\circ} 27$, Santo Domingo, Centro de Investigación y Acción Social de la Compañía de Jesús.

Dauhajre, A., J. Achecar y A. Swindale (1994): Estabilización, apertura y pobreza en República Dominicana, 1986-1992, Santo Domingo, Fundación Economía y Desarrollo.

Dauhajre, A., J. Aristy, E. Riley, R. Mena, S. Féliz, H. Guiliani y J. De Castro (1996): Programa macroeconómico de mediano plazo para la República Dominicana: 1996-2000, Santo Domingo, Editora Corripio.
Dauhajre, A., E. Riley, R. Mena y J. A. Guerrero (1989): Impacto económico de las zonas francas industriales de exportación de la República Dominicana, Santo Domingo, Fundación Economía y Desarrollo.

De Peña, J. (1977): Breve historia del movimiento sindical dominicano, Santo Domingo, Ediciones Dominicanas Populares.

Del Rosario, G. y T. Hidalgo (1986): Metodología para calcular el índice de salario nominal y su aplicación en la economía dominicana, Santo Domingo, Banco Central de la República Dominicana.

Duarte, I. (1979): La fuerza de trabajo infantil en Santo Domingo, Estudios sociales, $\mathrm{N}^{\circ} 12$, Santo Domingo, Centro de Investigación y Acción Social de la Compañía de Jesús.

(1983): Fuerza laboral urbana en Santo Domingo, 19801983, Estudios sociales, № 16, Santo Domingo, Centro de Investigación y Acción Social de la Compañía de Jesús.

Duarte, I., R. Brea y R. Tejada (1998): Cultura política y democrática en la República Dominicana, resultados de la segunda encuesta nacional de cultura política y democrática (DEMOS97), Santo Domingo, Pontificia Universidad Católica Madre y Maestra/Programa de Apoyo a las Iniciativas Democráticas/Asociación Probienestar de la Familia (PROFAMILIA)/Instituto de Estudios de Población y Desarrollo (IEPD), marzo.

Duarte, I., C. J. Gómez y M. Ariza (1991): Menores en circunstancias especialmente difíciles en la República Dominicana, Santo Domingo, Fondo de las Naciones Unidas para la Infancia (UNICEF)/PROFAMILIA/IEPD.

Education Development Center (1975): Los recursos humanos y el empleo en la República Dominicana. Informe y recomendaciones, Newton, Massachusetts.

FUNDAPEC (Fundación APEC de Crédito Educativo) (1992): Encuesta nacional de mano de obra ENMO-91, Santo Domingo.

García, N. E. y M. Valdivia (1985): Crisis externa, ajuste interno y mercado de trabajo: República Dominicana, 1980-83, Monografía sobre empleo, $N^{\circ} 49$, Santiago de Chile, Organización Internacional del Trabajo (OIT), Programa Regional del Empleo para América Latina y el Caribe (PREALC).

Gregory, P. (1997): Empleo y desempleo en la República Dominicana, Santo Domingo, Banco Central de la República Dominicana, diciembre.

Gurak, D. T., M. M. Kritz, V. Mota y M. Ortega (1979): Female employment in the Dominican Republic, trabajo presentado en la Population Association of America Meetings, Filadelfia, 26 de abril.

Harberger, A. C. (1997): Economic policy and performance in the Dominican Republic, Santo Domingo, noviembre.

Harris, J. y M. Todaro (1970): Migration, unemployment and development: A two-sector analysis, The American Economic Review, vol. 60, $\mathrm{N}^{\circ}$ 1, Nashville, Tennessee, American Economic Association.

Hernández, L. (1998): La negociación colectiva en Santo Domingo, Santo Domingo, Fundación Institucionalidad y Justicia (FINJUS).

Hernández, L. y C. Hernández, eds. (1996): La seguridad social en Santo Domingo, Santo Domingo, Instituto de Estudios del Trabajo, Inc./Editora Corripio.

Listín Diario (1999): Santo Domingo, 18 de agosto.

Liz, R. E. (1993): Crecimiento económico, empleo y capacitación, trabajo presentado al seminario Políticas de concertación para la reforma social y disminución de la pobreza, Santo Domingo, Programa de las Naciones Unidas para el Desarrollo (PNUD)/PUCMM, 23 y 24 de noviembre, mimeo.

Lizardo, J. (1994): Condiciones de los menores en la República Dominicana, Santo Domingo, IEPD/UNICEF, octubre.

Lizardo, M. M. y R. M. Guzmán (1999): Niveles de escolaridad y sus factores determinantes: una cuantificación econométrica, Ciencia y sociedad, vol.24, Santo Domingo, Instituto Tecnológico de Santo Domingo, abril-junio. 
Lozano, W. (1987): Desempleo estructural, dinámica económica y fragmentación de los mercados de trabajo urbanos: el caso dominicano, Ciencia y sociedad, vol. 12, $\mathrm{N}^{\circ} 3$, Santo Domingo, Instituto Tecnológico de Santo Domingo.

Martí, A. (1997): Instrumental para el análisis de la economía dominicana. Base de datos (1947-1995), Santo Domingo, Editora Búho.

Martínez, L. (1994): Actitudes femeninas frente a los oficios no tradicionales, Santo Domingo, CE-MUJER/ BID/FUNDAPEC.

Martínez, S. (1999): From hidden hand to heavy hand: Sugar, the estate, and migrant labor in Haiti and the Dominican Republic, Latin American Research Review, vol. 34, N 1, Yale, University of New Mexico.

Márquez, G. (1998): El desempleo en América Latina y el Caribe a mediados de los años 90, Documento de trabajo, $\mathrm{N}^{\circ} 377$, Washington, D.C., BID, Oficina del Economista Jefe, agosto.

Moya, F. (ed.) (1986): Condiciones socioeconómicas de la mujer trabajadora en la República Dominicana, Forum, $\mathrm{N}^{\circ} 21$, Santo Domingo.

Moya, F. y M. Ortiz (1994): Microempresas y microempresarios en la República Dominicana, 1993-1994, Santo Domingo, FONDOMICRO.

(1995): Indicadores de las microempresas en la República Dominicana, 1994-1995, FONDOMICRO, Santo Domingo, R.D.

Nicasio, I. y J. De La Rosa (1998): Historia, metodología y organización de los censos en República Dominicana, 1920-1993, Santo Domingo, Editora Búho.

OIT (Oficina Internacional del Trabajo) (1975): Generación de empleo productivo y crecimiento económico: El caso de la República Dominicana, Ginebra.

OIT (Organización Internacional del Trabajo) (1998): Anuario de estadísticas del trabajo, $\mathrm{N}^{\circ} 57$, Ginebra.

ONAPLAN (Oficina Nacional de Planificación) (1968): Plataforma para el desarrollo económico y social de la República Dominicana, Santo Domingo.

(1974): Bases para formular una política de empleo en la República Dominicana, serie PLANDES, $\mathrm{N}^{\circ} 19$, Santo Domingo.

ONAPLAN/CESDEM/PROFAMILIA (Oficina Nacional de Planificación/ Centro de Estudios Sociales y Demográficos/Asociación Probienestar de la Familia)(1997): Encuesta demográfica y de salud 1996, Santo Domingo.

ONE (Oficina Nacional de Estadística)(1993): Boletín de registro general de organizaciones sindicales en la República Dominicana, Santo Domingo.

(1998): Censo de servidores públicos, Santo Domingo.

Ortiz, M. (1996): Perfil de las microempresas en la República Dominicana y características de las microempresas de Santiago, 1995-1996, Santo Domingo, FONDOMICRO.

(1998): Microempresas y fuerza laboral en la República Dominicana, 1997-1998, Santo Domingo, FONDOMICRO.

Pérez Sáinz, J. P. (1995a): Actores y cultura laboral en la industria de exportación: una comparación entre Costa Rica y la República Dominicana, Documento de trabajo, $N^{\circ} 6$, San José de Costa Rica, OIT.

(1995b): Impacto sociolaboral de maquilas y zonas francas en Centroamérica y la República Dominicana, Documento de trabajo, $\mathrm{N}^{\circ} 16$, San José de Costa Rica, OIT.

PUCMM/CUEPS (Pontificia Universidad Católica Madre y Maestra/ Centro Universitario de Estudios Políticos y Sociales) (1996): Agenda nacional de desarrollo. Planes de acción para las diez principales prioridades, vol. II, Santo Domingo, GAD, Editora Centenario.

Quiterio, G. y J. Polanco (1990): República Dominicana: estimaciones y proyecciones de la población económicamente acti- va y demanda de empleos por regiones y subregiones, 19802000, Santo Domingo, ONAPLAN/IEPD, octubre.

Rama, M. (1995): Issues and non-issues in the Jamaican labor market, Washington, D.C., Banco Mundial.

Ramírez, N. (1993): La fuerza de trabajo en la República Dominicana: análisis de los resultados del cuestionario de hogar ampliado, encuesta demográfica y de salud 1991, ENDESA 1991, Santo Domingo, IEPD.

Ramírez, N., A. Tatis y D. Germán (1983): Población y mano de obra en la República Dominicana: perspectivas de la fuerza de trabajo y del empleo-desempleo en el período 1980-1990, Santo Domingo, IEPD.

República Dominicana, Secretaría de Estado de Trabajo (1997): Estudio sobre salario en la economía dominicana, Santo Domingo, Ecocaribe S.A., mayo.

(1999): Estadísticas laborales, Boletín $\mathrm{N}^{\circ} 4$, Santo Domingo, marzo.

República Dominicana, Secretaría de Estado de Turismo (1996): Turismo en cifras, Santo Domingo, julio.

Rodríguez, M. (1999): República Dominicana: los migrantes y el futuro del país, Listín diario, Santo Domingo, 24 de septiembre.

Santana, I. (1990): Informe sobre la mano de obra rural, Santo Domingo, Consejo Nacional de Agricultura, Proyecto Análisis de Política Agropecuaria, Unidad de Estudios Agropecuarios. (1996): Estrategias y políticas de lucha contra la pobreza y generación de empleo, PUCMM/CUEPS, Agenda nacional de desarrollo. Planes de acción para las diez principales prioridades, vol.II, Santo Domingo, GAD, Editora Centenario.

Santana, I. y M. Rathe (1993): Reforma social, una agenda para combatir la pobreza, Santo Domingo, Ediciones de la Fundación Siglo 21.

Santos, A. U. (1998): Análisis de los efectos de la educación secundaria en el individuo y el rol representado en el mercado de trabajo, Santo Domingo, Banco Mundial, mimeo.

Senado de la República Dominicana (1999): Proyectos de ley de seguridad social, documento base para las vistas públicas provinciales y del Distrito Nacional, Santo Domingo, Comisión Permanente de Seguridad Social, enero.

Silié, R. (1996): El trabajo infanto-juvenil en las ciudades dominicanas, Santo Domingo, Facultad Latinoamericana de Ciencias Sociales (FLACSO)/UNICEF.

Tejada, R., J. Herold y L. Morris (1998): República Dominicana: encuesta nacional de jóvenes 1992 ENJOVEN-92. Informe final, Santo Domingo, IEPD.

The CUNY Dominican Studies Institute (1997): Dominican Studies: Resources and Research Questions, Dominican research monographs, Nueva York.

USAID (Agencia de los Estados Unidos para el Desarrollo Internacional)/República Dominicana, Secretaría de Estado de Agricultura (1977): Empleo, República Dominicana. Serie de documentos sobre análisis sectorial agropecuario, Documento estadístico de trabajo $\mathrm{N}^{\circ} 1$, Washington, D.C.

Vargas, L. (1995): Fuerza de trabajo: desempleo, subempleo y salarios, documento presentado al seminario Problemas de Población en República Dominicana, Santo Domingo, ONAPLAN, Secretariado Técnico de la Presidencia, 31 de mayo al 2 de junio.

Weller, J. (1993): La situación del empleo en la República Dominicana, Informe de consultoría, San José de Costa Rica, OIT. (1998): Los mercados laborales en América Latina: su evolución en el largo plazo y sus tendencias recientes, Serie reformas económicas, $\mathrm{N}^{\circ} 11$, Santiago de Chile, Comisión Económica para América Latina y el Caribe (CEPAL), diciembre. 